\title{
Influence of dimensional static and dynamic charges on conduction in the active zone of a quantum cascade laser
}

\author{
A.M. Gryschuk ${ }^{1}$, I.V. Boyko \\ ${ }^{I}$ Ivan Franko Zhytomyr State University, \\ 40, Velyka Berdychivska str., Zhytomyr, phone: (0412) 372-763, e-mail: teor-caf@meta.ua \\ ${ }^{2}$ I. Pul'uj Ternopil National Technical University, \\ 56, Ruska str., Ternopil,e-mail: boyko.i.v.theory@gmail.com
}

\begin{abstract}
The theory of active dynamic conductivity in the three-barrier active zone of a quantum cascade laser has been developed in the model of the electron effective mass and rectangular potential in the low signal approximation. In the preceding paper, it was shown that the static charge causes an increase of the lifetime of electronic quasistationary states and the shift of the energy levels into the high-energy range without changing maximum values of the active dynamic conductivity. The dynamic charge causes redistribution of the partial components of the active dynamic conductivity without affecting the spectral parameters of electron. It has been set that the partial components of the dynamic conductivity caused by the passing through electron flow from nanostructures reduce, and the components of conductivity caused by the flow in the opposite direction increase, thus, the conductivity value remains constant.
\end{abstract}

Keywords: quantum cascade laser, resonant-tunnelling structure, dynamic conductivity, static charge, dynamic charge.

Manuscript received 04.11.14; revised version received 02.04.15; accepted for publication 27.05.15; published online 08.06.15.

\section{Introduction}

Nowadays, development of semiconductor technology and physics is closely related with researches in quantum cascade lasers (QCL) and detectors [1-8] as well as physical processes in them.

The active elements of the mentioned nanodevices operating in the terahertz and infrared region of electromagnetic field frequencies are flat resonanttunnelling structures (RTS), which physical and geometrical parameters greatly determine the properties of the mentioned nanodevices. So, to find out the conditions of nanolaser and detector optimization, it is important to know the essence of physical processes occurring at the coherent electron transport through the multilayer RTS. The theory of the electron transport through the three-barrier RTS with an applied permanent longitudinal electric field based on the founded selfconsistent solution of the full Schrödinger equation and Poisson equation has been developed in this paper.

Taking as an example a three-barrier RTS as an active zone of the experimentally realized QCL with $\mathrm{In}_{1-\mathrm{x}} \mathrm{Ga}_{\mathrm{x}} \mathrm{As}$ wells and $\mathrm{In}_{1-\mathrm{x}} \mathrm{Al}_{\mathrm{x}} \mathrm{As}$ barriers shows the effect of the dimensional static and dynamic charge on spectral parameters of quasi-stationary states (QSS) of an electron and an active dynamic conductivity of nanosystems.

\section{The theory of dynamic conduction in the three- barrier active zone of quantum cascade lasers}

To calculate the active dynamic conduction of electrons in the three-barrier active zone of QCL, let us consider that the system is placed in the Cartesian coordinate 
system in such a manner that its layers are perpendicular to the separation boundaries of nanosystems. The constant electric field $\vec{F}$ is applied along the normal to the layers of RTS. The geometric parameters of the system are known (Fig. 1).

As the difference between the lattice constant at the interfaces layer-well and layer-barrier is negligible, the model of an effective mass and rectangular potentials can be applied to electrons:

$$
\begin{aligned}
& m(z)=m_{w}\left[\theta(-z)+\sum_{p=1}^{3}\left(\theta\left(z-z_{2 p-1}\right)-\theta\left(z-z_{2 p}\right)\right)\right]+ \\
& +m_{b} \sum_{p=0}^{2}\left[\theta\left(z-z_{2 p}\right)-\theta\left(z-z_{2 p+1}\right)\right], \\
& U(z)=U \sum_{p=0}^{2}\left[\theta\left(z-z_{2 p}\right)-\theta\left(z-z_{2 p+1}\right)\right]- \\
& -e F\left\{z\left[\theta(z)-\theta\left(z-z_{5}\right)\right]+z_{5} \theta\left(z-z_{5}\right)\right\},
\end{aligned}
$$

where $\theta(z)$ is the Heaviside function; $z_{-1} \rightarrow-\infty, z_{6} \rightarrow \infty ; m_{w}$ and $m_{b}$ are the effective masses of electron in the potential wells and in barriers of the nanostructure.

It is considered that the monochromatic electron flow with the energy $E$ and initial concentration $n_{0}$ falls along the normal to the layers of open RTS on the left. The electron flow is considered to be one-dimensional and can be described by the wave function $\Psi(z, t)$, satisfying the full Schrödinger equation.

$$
\begin{aligned}
& i \hbar \frac{\partial \Psi(z, t)}{\partial t}= \\
& =\left(-\frac{\hbar^{2}}{2} \frac{\partial}{\partial z} \frac{1}{m(z)} \frac{\partial}{\partial z}+U(z)+H(z, t)\right) \Psi(z, t),
\end{aligned}
$$

where

$H(z, t)=-e \epsilon\left[z \theta(z)+\left(z_{5}-z\right) \theta\left(z-z_{5}\right)\right] \times$

$\times\left(e^{i \omega t}+e^{-i \omega t}\right)+e \varphi(z, t)$

is the Hamiltonian, which first summand describes interaction of electron with alternating electromagnetic field with the frequency $\omega$ and the tension amplitude of its electric component $\epsilon$. The second summand describes interaction of electron with the dimensional space charge, which potential $\varphi(z, t)$ can be found from the Poisson equation:

$\frac{\partial}{\partial z}\left[\varepsilon(z) \frac{\partial \varphi(z, t)}{\partial z}\right]=-4 \pi e n(z, t)$,

where

$$
\begin{aligned}
& \varepsilon(z)=\varepsilon_{w}\left\{\theta(-z)+\sum_{p=1}^{3}\left[\theta\left(z-z_{2 p-1}\right)-\theta\left(z-z_{2 p}\right)\right]\right\} \\
& +\varepsilon_{b} \sum_{p=0}^{2}\left[\theta\left(z-z_{2 p}\right)-\theta\left(z-z_{2 p+1}\right)\right]
\end{aligned}
$$

is the dielectric permittivity of three-barrier RTS, $\varepsilon_{w}$ and $\varepsilon_{b}$ are dielectric permittivity of layer materials in potential wells and barriers, and

$n(z, t)=n_{0}|\Psi(z, t)|^{2}$

is the variable in the electron density space.

It is evident from the structure of equations (3) and (5), considering the Hamiltonian form (4) and the equation (7), that they form a self-consistent system.

The solution of the full Schrödinger equation (3) with the Hamiltonian (4) in the weak signal approximation looks like:

$$
\begin{aligned}
& \Psi(z, t)=\Psi_{0}(z) e^{-i \omega_{0} t}+\Psi_{-1}(z) e^{-i\left(\omega_{0}-\omega\right) t}+ \\
& +\Psi_{+1}(z) e^{-i\left(\omega_{0}+\omega\right) t}, \quad \omega_{0}=E / \hbar .
\end{aligned}
$$

Substituting the equivalence (7) in the Poisson equation (5), considering (8), with keeping the summands of the first degree, we have got the following equation:

$$
\begin{aligned}
& \frac{\partial}{\partial z}\left[\varepsilon(z) \frac{\partial \varphi(z, t)}{\partial z}\right]= \\
& =-4 \pi e n_{0}\left[\xi_{0}(z)+\xi(z) e^{i \omega t}+\xi^{*}(z) e^{-i \omega t}\right],
\end{aligned}
$$

where

$$
\begin{aligned}
& \xi_{0}(z)=\left|\Psi_{0}(z)\right|^{2}, \\
& \xi_{+}(z)=\Psi_{0}(z) \Psi_{+1}^{*}(z)+\Psi_{0}^{*}(z) \Psi_{-1}(z), \\
& \xi_{-}(z)=\xi_{+}^{*}(z) .
\end{aligned}
$$

For any $p$-layers from RTS, the solution of the equation (9) looks like that:

$$
\begin{aligned}
& \varphi(z, t)=\sum_{p=1}^{5}\left[\varphi_{s t}^{(p)}(z)+\varphi_{-}^{(p)}(z) e^{i \omega t}+\varphi_{+}^{(p)}(z) e^{-i \omega t}\right] \times \\
& \times\left[\theta\left(z-z_{p-1}\right)-\theta\left(z-z_{p}\right)\right] .
\end{aligned}
$$

The following equations (12), (13) are got from (9), considering (11) after equating the values of the same order of smallness.

$$
\begin{aligned}
& \frac{\partial^{2} \varphi_{s t}^{(p)}(z)}{\partial z^{2}}=-\frac{4 \pi e n_{0}}{\varepsilon_{p}} \xi_{0}^{(p)}(z), \\
& \frac{\partial^{2} \varphi_{ \pm}^{(p)}(z)}{\partial z^{2}}=-\frac{4 \pi e n_{0}}{\varepsilon_{p}} \xi_{\mp}^{*(p)}(z),
\end{aligned}
$$

and its solutions are as follows:

$$
\begin{aligned}
& \varphi_{s t}^{(p)}(z)=-\frac{4 \pi e n_{0}}{\varepsilon_{p}} \times \\
& \times \int_{0}^{z} \int_{0}^{z_{1}} \xi_{0}^{(p)}\left(z_{2}\right) d z_{1} d z_{2}+C_{1}^{(p)}\left(z-z_{p-1}\right)+C_{2}^{(p)}, \\
& \varphi_{ \pm}^{(p)}(z)=-\frac{4 \pi e n_{0}}{\varepsilon_{p}} \int_{0}^{z} \int_{0}^{z_{1}} \xi_{\mp}{ }^{(p)}\left(z_{2}\right) d z_{1} d z_{2}+ \\
& +C_{1}^{\mp(p)}\left(z-z_{p-1}\right)+C_{2}^{\mp(p)} .
\end{aligned}
$$


They determine the potential $\varphi_{s t}^{(p)}(z)$ caused by the static space charge and potentials determined by the dimensional dynamic charge in the case of electronic transition with the absorption of $\varphi_{+}^{(p)}(z)$ and emission of photons $\varphi_{-}^{(p)}(z)$.

All the unknown coefficients $C_{1}^{(p)} ; C_{2}^{(p)} ; C_{1}^{\mp(p)} ; C_{2}^{\mp(p)}$ are uniquely determined from the continuity conditions of the potential $\varphi_{p}(z, t)$ and electric displacement field within all RTS.

Substituting (11) and (8) from the full Schrödinger equation (5), after equating the coefficients of $e^{ \pm i \omega t}$ to the variables of the zero degree, we have got the equation:

$\left(-\frac{\hbar^{2}}{2} \frac{\partial}{\partial z} \frac{1}{m(z)} \frac{\partial}{\partial z}+U(z)+e \varphi_{s t}(z)-E\right) \Psi_{0}(z)=0$,

$\left(-\frac{\hbar^{2}}{2} \frac{\partial}{\partial z} \frac{1}{m(z)} \frac{\partial}{\partial z}+U(z)+e \varphi_{s t}(z)-\hbar\left(\omega \pm \omega_{0}\right)\right) \Psi_{ \pm 1}(z)=$

$\left.=e^{(a}\left[z \theta(z)+\left(z_{5}-z\right) \theta\left(z-z_{5}\right)\right]-\varphi_{ \pm}(z)\right) \Psi_{0}(z)$.

The resulting Schrödinger equations (16), (17) together with the Poisson equations (12), (13) form a set of mutually agreed equations.

The solutions of them are as follows:

$\Psi_{ \pm 1}^{0}(z)=\Psi_{0 \pm}(z)+\Phi_{0 \pm}(z)$.

Here,

$\Psi_{0 \pm}(z)=B_{0 \pm}^{(0)} e^{-i k_{ \pm}^{(0)} z} \theta(-z)+A_{0 \pm}^{(6)} e^{i k_{ \pm}^{(6)}\left(z-z_{5}\right)} \theta\left(z-z_{5}\right)+$

$+\sum_{p=1}^{4} \sum_{l=1}^{N}\left[A_{0 \pm}^{(p, l)} e^{i K_{ \pm}^{(p, l)}\left(z-z_{l-1}\right)}+B_{0 \pm}^{(p, l)} e^{-i K_{ \pm}^{(p, l)}\left(z-z_{l-1}\right)}\right] \times$

$\times\left[\theta\left(z-z_{l-1}\right)-\theta\left(z-z_{l}\right)\right]$,

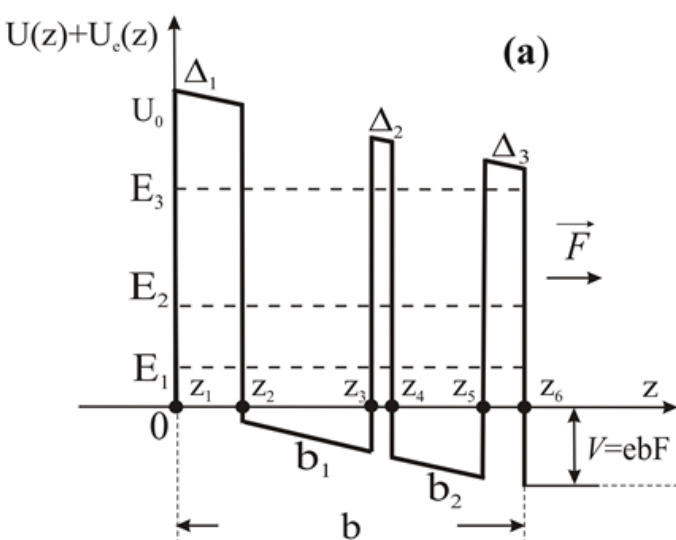

$\Phi_{0 \pm}(z)=\sum_{p=1}^{4} \sum_{l=1}^{N}\left(\mp \frac{e \epsilon z}{\hbar \omega} \Psi_{0}^{(6)}(z)+\frac{e \epsilon}{m_{l} \omega^{2}} \frac{d \Psi_{0}^{(l)}(z)}{d z}\right) \times$

$\times\left[\theta\left(z-z_{l-1}\right)-\theta\left(z-z_{l}\right)\right]+\frac{e € z_{5}}{\hbar \omega} \Psi_{0}^{(6)}(z) \theta\left(z-z_{5}\right)$,

where

$k_{ \pm}^{(0)}=k_{ \pm}^{(6)}=\hbar^{-1} \sqrt{2 m_{0}\left(\hbar\left(\omega_{0} \pm \omega\right)\right)} ;$

$K_{ \pm}^{(p, l)}=K_{ \pm}^{(p, l)}\left(z_{l}\right)=\left\{\begin{array}{c}\sqrt{2 m_{0}\left(\hbar\left(\omega_{0} \pm \omega\right)-e \varphi\left(z_{l}\right)\right)}, \\ \text { in the barrier } \\ \sqrt{2 m_{1}\left(\hbar\left(\omega_{0} \pm \omega\right)-U-e \varphi\left(z_{l}\right)\right)} . \\ \text { out the barrier }\end{array}\right.$

The formula to calculate the electric currents density through RTS is as follows:

$$
\begin{aligned}
& j(E \pm \hbar \omega, z)=\frac{i e \hbar n_{0}}{2 m_{w}}\left(\Psi_{ \pm 1}(E, z) \frac{d \Psi_{ \pm 1}^{*}(E, z)}{d z}-\right. \\
& \left.-\Psi_{ \pm 1}^{*}(E, z) \frac{d \Psi_{ \pm 1}(E, z)}{d z}\right) .
\end{aligned}
$$

And it is proportional to the real parts of the corresponding active dynamic conductivities $\sigma^{ \pm}(E, \Omega)$.

The full active dynamic conductivity of RTS $\sigma(E, \Omega)$ can be determined as the sum of two partial components:

$\sigma(E, \Omega)=\sigma^{+}(E, \Omega)+\sigma^{-}(E, \Omega)$,

where

$$
\begin{gathered}
\sigma^{+}(E, \Omega)=\frac{\hbar \Omega n_{0}}{2 m_{0} z_{5}{ }^{a}{ }^{2}}\left(k_{+1}^{(6)}\left|A_{+1}^{(6)}\right|^{2}-k_{-1}^{(6)}\left|A_{-1}^{(6)}\right|^{2}\right), \\
\sigma^{-}(E, \Omega)=\frac{\hbar \Omega n_{0}}{2 m_{0} z_{5}{ }^{2}{ }^{2}}\left(k_{+1}^{(0)}\left|B_{+1}^{(0)}\right|^{2}-k_{-1}^{(0)}\left|B_{-1}^{(0)}\right|^{2}\right) .
\end{gathered}
$$

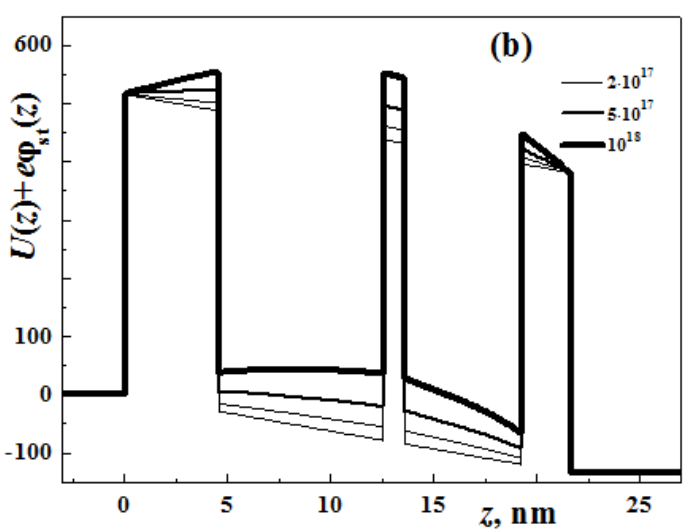

Fig. 1. Geometrical and energetic schemes of the three-barrier RTS $(a)$ and the renormalized potential profile of the three-barrier RTS, caused by the static charge from the dependence on $z$ with the electron energy $E=E_{3}^{s t}$ and charge carrier concentrations $n=2 \cdot 10^{17} \mathrm{~cm}^{-3}, 5 \cdot 10^{17}, 10^{18}(b)$.

(C) 2015, V. Lashkaryov Institute of Semiconductor Physics, National Academy of Sciences of Ukraine 


\section{Discussion of the results}

The calculation of the active dynamic conductivity and spectral parameters of quasi-stationary electronic states was done using the active zone of the experimental QCL as an example, which was described in the work [9] (Fig. 1). RTS contains $\operatorname{In}_{0.53} \mathrm{Ga}_{0.47}$ As wells and $\mathrm{In}_{0.52} \mathrm{Ga}_{0.48} \mathrm{As}$ barriers and can be described by the following geometrical $\Delta_{1}=4.5 \mathrm{~nm}, \Delta_{2}=1.0 \mathrm{~nm}, \Delta_{3}=$ $2.4 \mathrm{~nm}, b_{1}=8.0 \mathrm{~nm}, b_{2}=5.7 \mathrm{~nm}$ and physical $m_{w}=$ $0.041 m_{e}, m_{b}=0.082 m_{e}, U=516 \mathrm{meV}, \varepsilon_{w}=13.899, \varepsilon_{b}=$ 12.726 parameters. Fig. $1 \mathrm{~b}$ describes the potential profile of the studied RTS renormalized by the static charge that was calculated for different values of the concentration of electrons $\left(n=2 \cdot 10^{17} \mathrm{~cm}^{-3}, 5 \cdot 10^{17}, 10^{18}\right)$. It is obvious from Fig. 1b that the increase of electron concentration causes deformation of the potential profile of nanosystem, which influences spectral characteristics of electronic QSS. The mentioned changes reflect the results of the calculation of the transparency coefficient $D(E)$ (Fig. 2a) within the limits of energies of the first three QSS, the resonance energies $E_{n}^{s t}$, and the lifetimes of electrons $\tau_{n}^{s t}$ (Fig. 2a) depending on the concentration of the charge $n_{0}$, considering the space charge.

As seen from Fig. 2a, the dimensional charge deforms the Lorentz shape to the wedge shape, causing the increase in values of all resonant energies $\left(E_{n}^{\text {st }}\right)$ and widths $\left(\Gamma_{n}^{\text {st }}\right)$ (Fig. 2b). The values of the maxima of $D(E)$ with augmentation of the concentration increase. As the electron lifetimes $\left(\tau_{n}^{\mathrm{st}}\right)$ in the corresponding QSS (a)
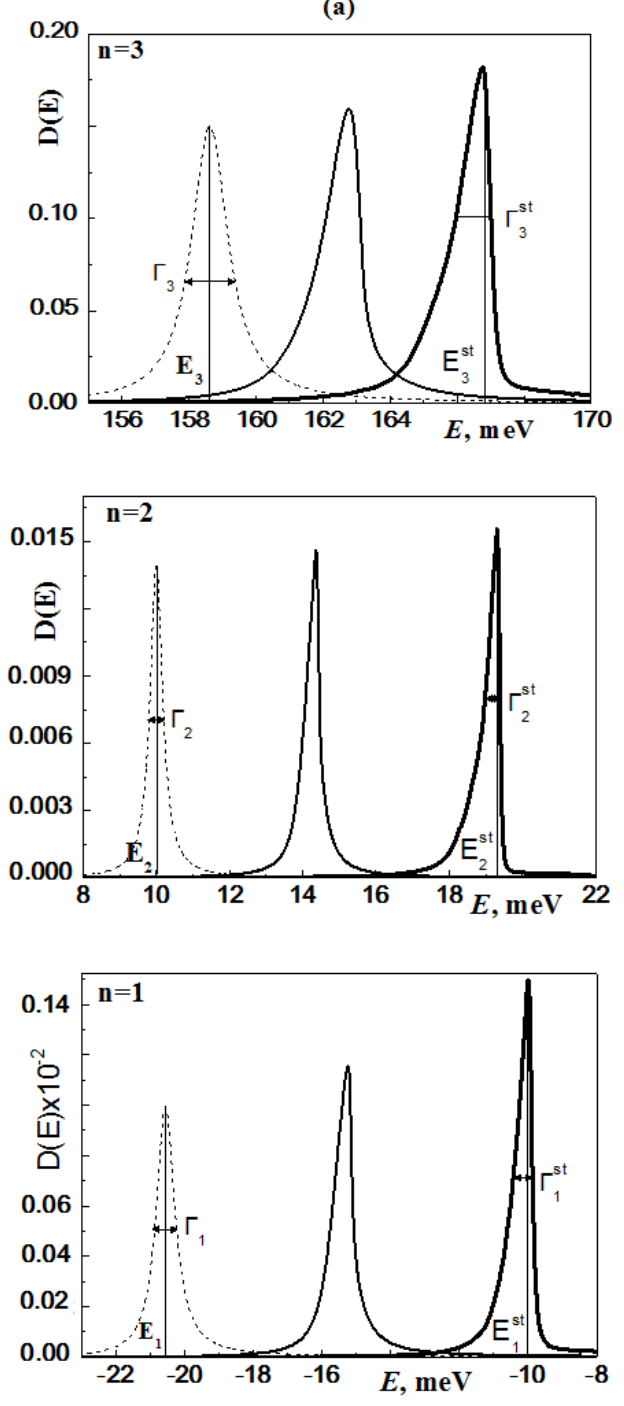

(b)
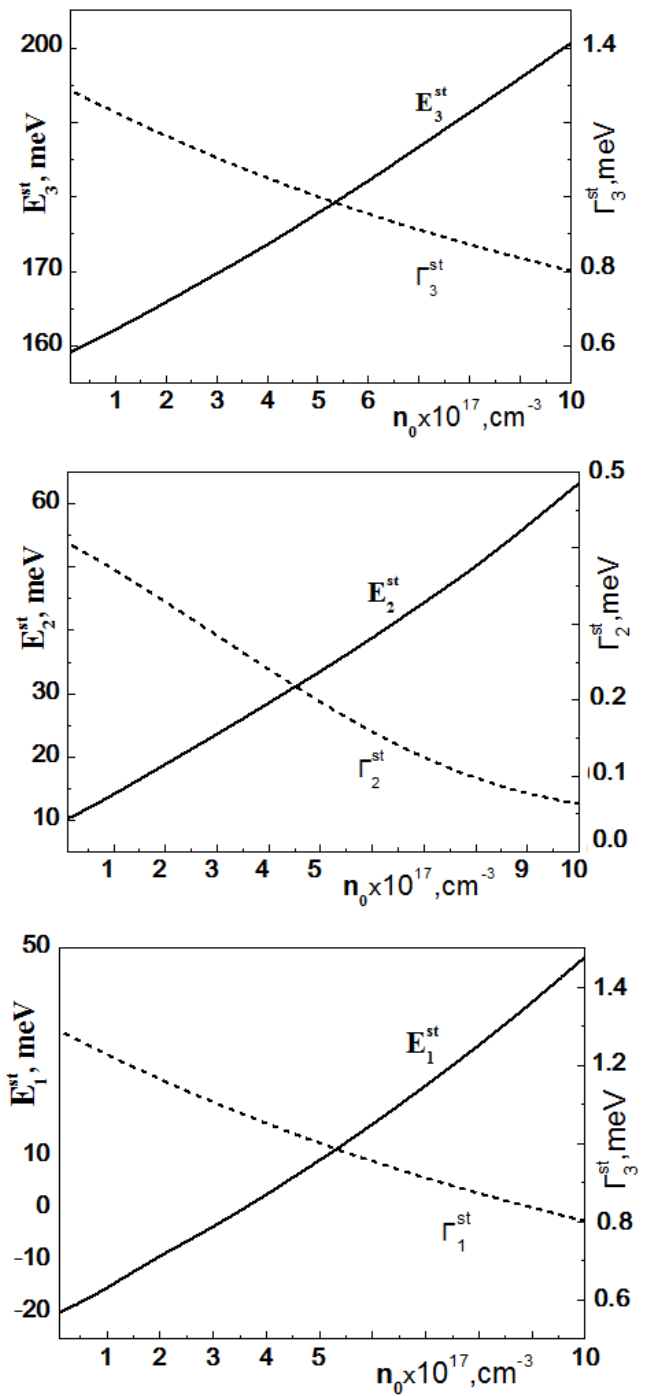

Fig. 2. Dependences of the transparency coefficient on the energy without (dotted line) and with the dimensional charge (solid lines) of the first three quasi-stationary states at concentrations $n_{0}=10^{17}, 2 \cdot 10^{17} \mathrm{~cm}^{-3}(a)$ and dependences of their resonance energies $\left(E_{1}^{\mathrm{st}}, E_{2}^{\mathrm{st}}, E_{3}^{\mathrm{st}}\right)$ and widths $\left(\Gamma_{1}^{\mathrm{st}}, \Gamma_{2}^{\mathrm{st}}, \Gamma_{3}^{\mathrm{st}}\right)$ on the concentration $n_{0}(b)$. 


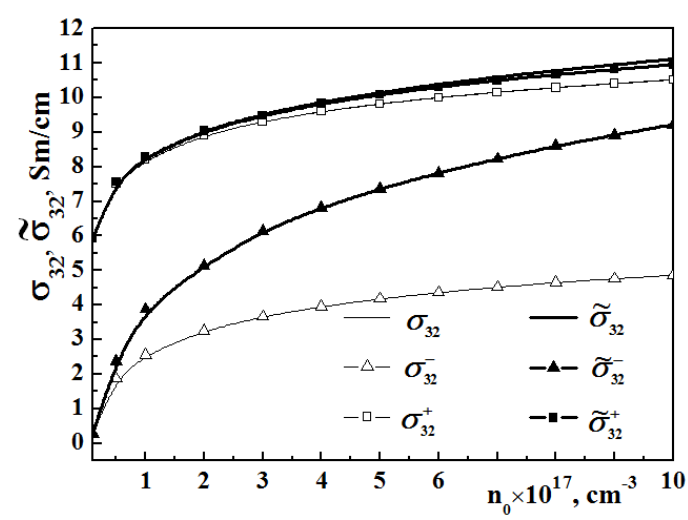

Fig. 3. Dependences of logarithms of dynamic conductivity, resulting in laser transitions $3 \rightarrow 2$ and their partial components, calculated considering the dimensional charge $\left(\tilde{\sigma}_{32}, \tilde{\sigma}_{32}^{+}, \tilde{\sigma}_{32}^{-}\right)$and without consideration $\left(\sigma_{32}, \sigma_{32}^{+}, \sigma_{32}^{-}\right)$, on the concentration of charge carriers $n_{0}$. are related with resonant widths $\left(\Gamma_{n}^{\mathrm{st}}\right)$, as $\tau_{n}^{\mathrm{st}}=\hbar / \Gamma_{n}^{\mathrm{st}}$, so the augmentation of electron concentration causes its incensement. And it is the significant factor for the great values of $n_{0}$.

Fig. 3 shows the results of calculating the logarithms of the dynamic conductivity appearing in the quantum transition $3 \rightarrow 2$ and its partial components without $\left(\sigma_{32}, \sigma_{32}^{+}, \sigma_{32}^{-}\right)$and considering $\left(\tilde{\sigma}_{32}, \tilde{\sigma}_{32}^{+}, \tilde{\sigma}_{32}^{-}\right.$) the influence of the dimensional charge.

Fig. 3 demonstrates that in the case of conductivity that was calculated excluding the influence of the dimensional charge, the value of the component of the conduction determined by the electron flow to the output from nanosystems $\left(\sigma^{+}\right)$is bigger than the component of the conductivity $\left(\sigma^{-}\right)$defined by the flow in the opposite direction, i.e. $\sigma_{32} \approx \sigma_{32}^{+} \gg \sigma_{32}^{-}$. From the calculated dependences of the dynamic conductivity with considering the dimensional charge, it is obvious that with increasing the concentration of electrons $n_{0}$, the total value of conductivity $\tilde{\sigma}_{32}$ increases and the partial conduction component, determined by the direct electron flow $\left(\tilde{\sigma}_{32}^{+}\right.$) decreases, but the component in the opposite direction $\left(\tilde{\sigma}_{32}^{-}\right.$) increases. Thus, the dimensional charge causes redistribution of partial components in the total value of conductivity with increasing the concentration.

\section{Conclusions}

The quantum-mechanical theory of the spectral parameters of quasi-stationary states and dynamic conductivity of three-barrier RTS with a constant electric field as an active zone of QCL, considering the variable dimensional charge appearing in the process of electron transport through the nanostructure in a variable electromagnetic field has been developed in this paper.
The self-consistent solution of the Schrödinger and Poisson equations for different random electrons has been obtained in the model of effective masses and rectangular potentials.

The calculation of resonant energies, resonance widths, active dynamic conductivity and its partial components in the nanosystem model that corresponds to the experimental values of QCL was made in this paper. The value of the resonant energies that correlates with the experimental data was calculated with the accuracy not less than 5\%. It has been shown that with incensement of the concentration of electrons, the energy of laser radiation in a quantum transition $3 \rightarrow 2$ decreases, and the total value of active dynamic conductivity increases, thus the contribution of the partial component of conductivity determined by flow and directed opposite to the exit of nanosystems increases in it.

\section{References}

1. J.M. Wolf, A. Bismuto, M. Beck, and J. Faist, Distributed-feedback quantum cascade laser emitting at $3.2 \mu \mathrm{m} / /$ Opt. Exp. 22(2), p. 2111-2118 (2014).

2. D. Bachmann, M. Rösch, C. Deutsch, M. Krall, G. Scalari, M. Beck, J. Faist, K. Unterrainer and J. Darmo, Spectral gain profile of a multi-stack terahertz quantum cascade laser // Appl. Phys. Lett. 105(18), 181118-1-181118-4 (2014).

3. A. Buffaz, M. Carras, L. Doyennette, A. Nedelcu, X. Marcadet and V. Berger, Quantum cascade detectors for very long wave infrared detection // Appl. Phys. Lett. 96(17), 172101-1-172101-3 (2010).

4. D. Hofstetter, F.R. Giorgetta, E. Baumann, Q. Yang, C. Manz and K. Kohler, Midinfrared quantum cascade detector with a spectrally broad response // Appl. Phys. Lett. 93(22), 221106-1221106-3 (2008).

5. M.V. Tkach, Ju.O. Seti, I.V. Boyko, Effect of nonlinear electron-electron interaction on electron tunneling through an asymmetric two-barrier resonance tunnel structure // Ukr. J. Phys. 57(8), p. 849-859 (2012).

6. Ju.O. Seti, M.V. Tkach, I.V. Boyko, Influence of non-linear electrons interaction at their transport through the symmetric two-barrier resonance nanosystem // J. Optoelectron. Adv. Mater. 14(3-4), p. 393-400 (2012).

7. X. Gao, D. Botez, and I. Knezevic, Phonon confinement and electron transport in GaAs-based quantum cascade structures // J. Appl. Phys. 103(7), 073101-1-073101-9 (2008).

8. M.V. Tkach, Ju.O. Seti, Ju.B. Grynyshyn, Influence of confined polarization phonons on the electron spectrum in the three-barrier active zone of a quantum cascade detector // Ukr. J. Phys. 59(12), p. 1191-1200 (2014).

9. C. Sirtori, J. Faist, F. Capasso, D. L. Sivco, Midinfrared $(8.5 \mu \mathrm{m})$ semiconductor lasers operating at room temperature // IEEE Photonics Technology Lett. 9(3), p. 294-296 (1997). 